\title{
PRECIPITAÇÃO E EVENTOS EXTREMOS: MAPEAMENTO BIBLIOMÉTRICO DE ARTIGOS CIENTÍFICOS INTERNACIONAIS
}

\author{
Denise Dias dos Santos ${ }^{(\mathrm{a})}$, Alfredo Pereira de Queiroz Filho ${ }^{(\mathrm{b})}$, Emerson Galvani $^{(\mathrm{c})}$ \\ (a) Departamento de Geografia/FFLCH, Universidade de São Paulo, denise.dias.santos@usp.br \\ (b) Departamento de Geografia/FFLCH, Universidade de São Paulo, aqueiroz@usp.br \\ (c) Departamento de Geografia/FFLCH, Universidade de São Paulo, egalvani@usp.br
}

Climatologia em diferentes níveis escalares: mudanças e variabilidades

\begin{abstract}
Resumo
Os eventos extremos de precipitação consistem em um dos principais agentes na deflagração de desastres naturais, principalmente em ambientes de clima tropical. Considerando que as suas características e circunstâncias são muito diversificadas, que o volume de publicações é expressivo e que o número de revistas científicas é relevante, entende-se que é fundamental adotar estratégias que permitam avaliar o panorama geral da produção acadêmica internacional sobre esse tema. Nesse sentido, o objetivo deste trabalho é realizar um mapeamento bibliométrico dos artigos internacionais sobre as precipitações, especificamente com foco nos eventos extremos, fazendo uso de palavraschave e apoiando-se na base de dados Web of Science Core Collection. Por meio da metodologia de acoplamento bibliográfico e co-citação, foram analisados 1770 artigos e elaborados mapas bibliográficos no software VOSviewer. O artigo mais citado ("Climate Extremes: Observations, Modeling, and Impacts") recebeu 1794 citações. Constatou-se que os clusters formados a partir das palavras-chaves mais utilizadas e os termos mais citados revelam sua interligação com a área de variabilidade e mudanças climáticas. Os periódicos em destaque na pesquisa foram o Journal of Hydrology e o International Journal Of Climatology.
\end{abstract}

Palavras chave: mapeamento bibliométrico; chuva; precipitação; eventos extremos.

\section{Introdução e objetivo}

Para o estudo de atributos climáticos em uma determinada localidade, é necessária a análise do contexto em que eles estão inseridos, não somente com características locais, mas também considerando a circulação geral da atmosfera. Em uma região tropical chuvosa, por exemplo, o atributo climático de maior relevância é a precipitação, que podem definir os períodos de secas e de chuvas (GALVANI et al., 1998).

Em regiões tropicais e litorâneas, fatores como a latitude, o relevo e a alta umidade que se desloca do oceano para o continente podem interferir no clima local. Tal configuração proporciona uma distribuição das chuvas peculiar, em que não é possível estabelecer uma estação seca e há a ocorrência de eventos extremos. Estes eventos podem ocorrer em várias escalas de tempo, de diária a mensal e sazonal (CAVALCANTI, 2012). As inundações e escorregamentos ocorrem quando, principalmente em área urbana, os excessos de chuvas (relacionados a células convectivas profundas, muitas vezes embutidos em 
XVII Simpósio Brasileiro

de Geografia Física Aplicada

I Congresso Nacional

de Geografia Física
OS DESAFIOS DA GEOGRAFIA FÍSICA NA FRONTEIRA DO CONHECIMENTO

Instituto de Geociências - Unicamp

Campinas - SP

28 de Junho à 02 de Julho de 2017

sistemas sinóticos que podem persistir sobre a área) são combinados com alta impermeabilização do solo e ocupação em áreas de alta susceptibilidade aos escorregamentos, causando perdas e danos não somente materiais, mas também humanos.

A bibliometria é considerada um procedimento de estudo confiável para mensurar a produção científica de um determinado setor ou área da ciência. Já o mapeamento bibliométrico é uma metodologia que possibilita a representação gráfica da pesquisa realizada e visa o mapeamento da literatura relevante, que pode apresentar uma multiplicidade de dados quantitativos. Com o mapeamento bibliométrico, é possível definir o incremento da produção científica sobre determinado tema ou assunto, conhecer os principais periódicos, instituições e autores, bem como seus impactos nas citações de trabalhos produzidos na área. A análise bibliométrica é uma importante reflexão que pode colaborar na tomada de decisões de caráter estratégico; também é importante para que o pesquisador ou um centro de pesquisa identifique os periódicos mais relevantes na sua categoria, em função da sua visibilidade internacional definida pela sua posição nos rankings mundiais e/ou fator de impacto, por exemplo, e proporciona o estabelecimento de redes de colaboração com as instituições mais produtivas e de maior qualidade científica baseada em suas publicações (ROJAS-SOLA; AGUILERA-GARCIA, 2014).

Em um estudo bibliométrico realizado com o uso da palavra-chave "energia hidrelétrica", Jiang et al. (2016) afirmam que as conclusões da pesquisa bibliométrica podem colaborar para a futura política energética; as análises apontam que crescimento rápido das publicações indica que há uma enorme demanda por pesquisas relacionadas à energia hidrelétrica. E, juntamente com o processo acelerado de desenvolvimento hidrelétrico, os governos deveriam fornecer mais financiamento para este campo de pesquisa.

Haunschild et al. (2016) realizaram um estudo bibliométrico dedicado às pesquisas relacionadas às mudanças climáticas, que mostra um forte aumento das publicações sobre esta temática entre os anos de 1980 e 2014. Segundo os autores, os termos "vulnerabilidade" e "adaptação" aparecem em maior proporção em artigos considerados importantes (com maior número de citações); nota-se também que a temática de mudanças climáticas tornou-se importante para outras áreas do conhecimento/especialidades além da Climatologia e Meteorologia, com aumento expressivo nas Ciências Sociais e Engenharias, por exemplo. 
As palavras-chave ${ }^{1}$ são um importante item das publicações que auxiliam na identificação do conteúdo central; por meio das palavras-chave, pode-se definir o desenvolvimento e as tendências de uma determinada análise bibliométrica (ZHANG et al, 2016).

Nesse sentido, o objetivo deste trabalho é realizar um mapeamento bibliométrico dos artigos internacionais sobre as precipitações, especificamente com foco nos eventos extremos, com o uso de palavras-chave.

\section{Metodologia}

Para fins de análise e realização do mapeamento bibliométrico, serão utilizadas as técnicas de acoplamento bibliográfico e co-citação. $\mathrm{O}$ acoplamento entre dois artigos acontece quando estes fazem referência, no mínimo, um artigo em comum, estabelecendo uma conexão entre os dois trabalhos. Já a cocitação determina a "ligação/semelhança de dois documentos citados, via suas frequências de ocorrência conjunta em uma lista de referências dos autores citantes." (GRÁCIO, 2016, p.88). O acoplamento não se altera com o tempo, possui frequência fixa e permite avaliação retrospectiva. Em contrapartida a cocitação não é fixa, podendo aumentar com o tempo.

Este trabalho baseia-se, também, na metodologia utilizada por Zhang et al. (2016). Esses autores realizaram um mapeamento bibliométrico sobre o "imposto de carbono", a partir do critério da coocorrência de palavras-chave. Segundo os autores, "a ocorrência de duas palavras-chave pode expressar a forma na qual um determinado assunto é abordado em uma pesquisa". Os autores mencionam que "quando há relação interna entre estas duas palavras, quanto maior o número de ocorrências, mais próximas serão seu relacionamento e distância no texto" ${ }^{2}$.

Para a estruturação deste trabalho, foram utilizadas:

- Combinação de palavras-chaves: "rainfall" and "extreme events" or "precipitation" and "extreme events";

- Dados da plataforma Web of Science Core Collection (WoS - https://webofknowledge.com/);

- Software VOSViewer (http://www.vosviewer.com/).

A metodologia do mapeamento bibliométrico foi utilizada para a elaboração das análises e representações bibliométricas, com ênfase nas áreas de pesquisa, periódicos, autores e termos mais citados

\footnotetext{
${ }^{1}$ Segundo a norma NBR 6028 da Associação Brasileira de Normas Técnicas (ABNT, 2003c), as palavras-chave são palavras representativas do conteúdo do documento, escolhidas, preferentemente, em vocabulário controlado.

${ }^{2}$ Tradução livre dos autores.
} 
(HAUNSCHILD et al.,2016; WALTMAN et al., 2010). A figura 1 ilustra as etapas desenvolvidas no trabalho.

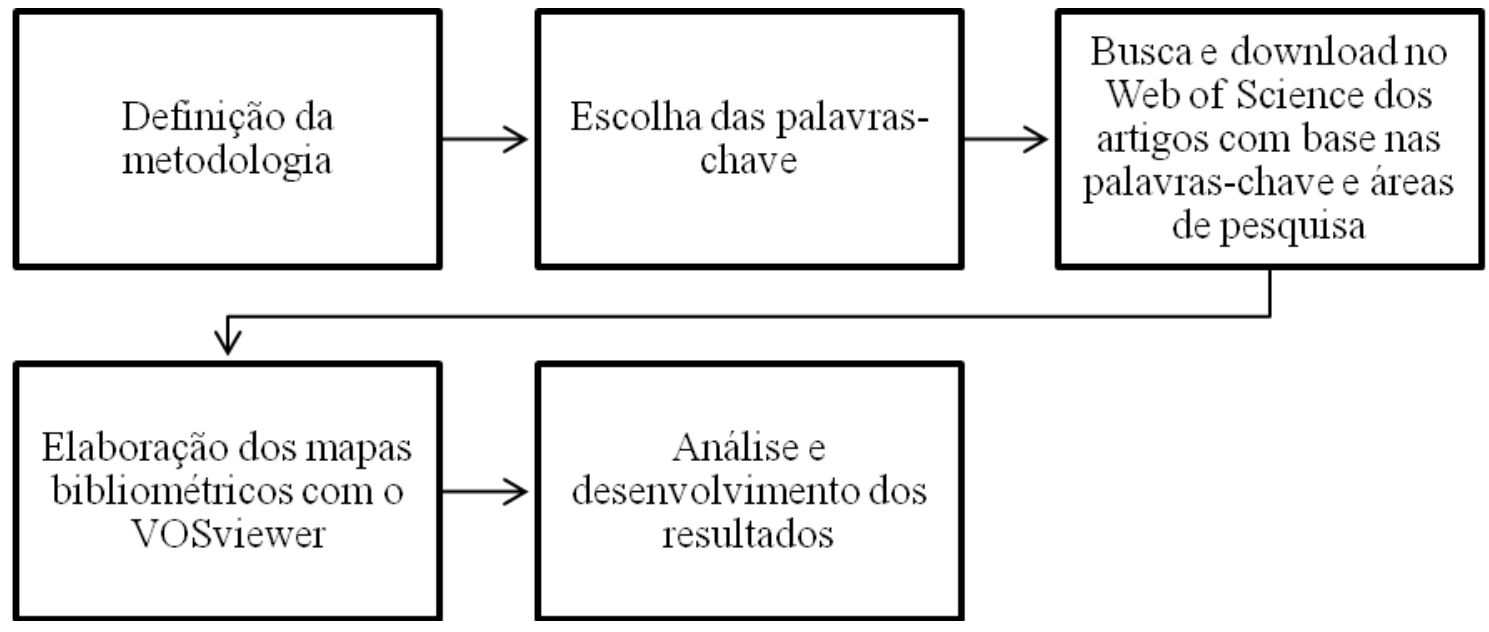

Figura 1: Fluxograma com as etapas desenvolvidas no trabalho. Organizado por: Santos, 2017.

\section{Resultados e Discussão}

A busca desenvolvida na plataforma Web of Science gerou um total de 1770 artigos pertinentes à pesquisa.

Por meio do acoplamento bibliométrico, foram gerados os mapas de artigos e periódicos. No primeiro (artigos), têm-se as referências compartilhadas por dois artigos, e quanto maior o número, maior é a similaridade de temas e área de pesquisa; neste mapa os artigos foram separados em cinco clusters (figura 2). 


\section{绐 VOSviewer}

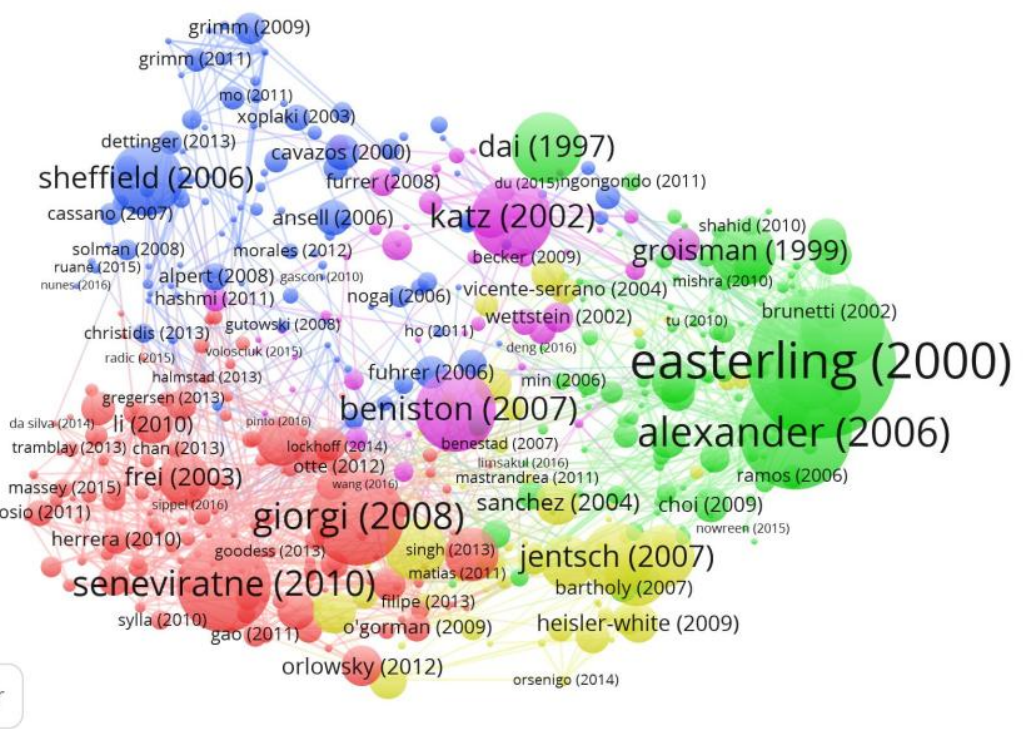

Figura 2: Mapa de acoplamento bibliométrico de artigos. Fonte: Web of Science, 2017.

O acoplamento de periódicos também permitiu definir aqueles que possuem afinidade de temas abordados em seus conteúdos (figura 3). Os clusters definem os agrupamentos por temas: em vermelho, periódicos ligados à hidrologia e processos hidrológicos; em verde, os ligados aos processos geomorfológicos, em lilás estão os relacionados às ciências atmosféricas; em amarelo, os que tratam de temas de meteorologia/climatologia e, por fim, em azul os relacionados às mudanças climáticas. De acordo com o mapeamento, os que mais se destacam é o Journal Of Hydrology, seguido pelo International Journal Of Climatology. 


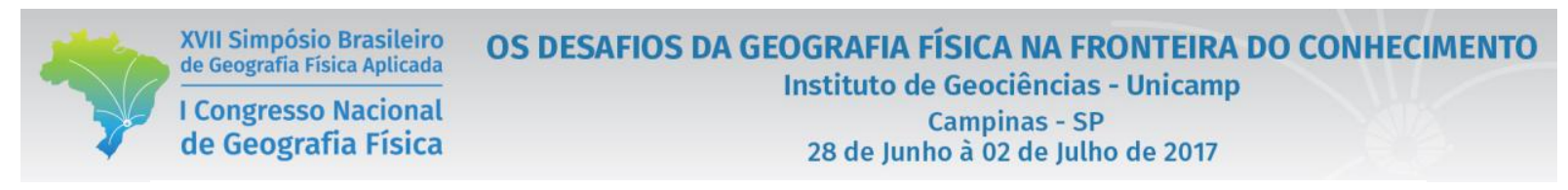

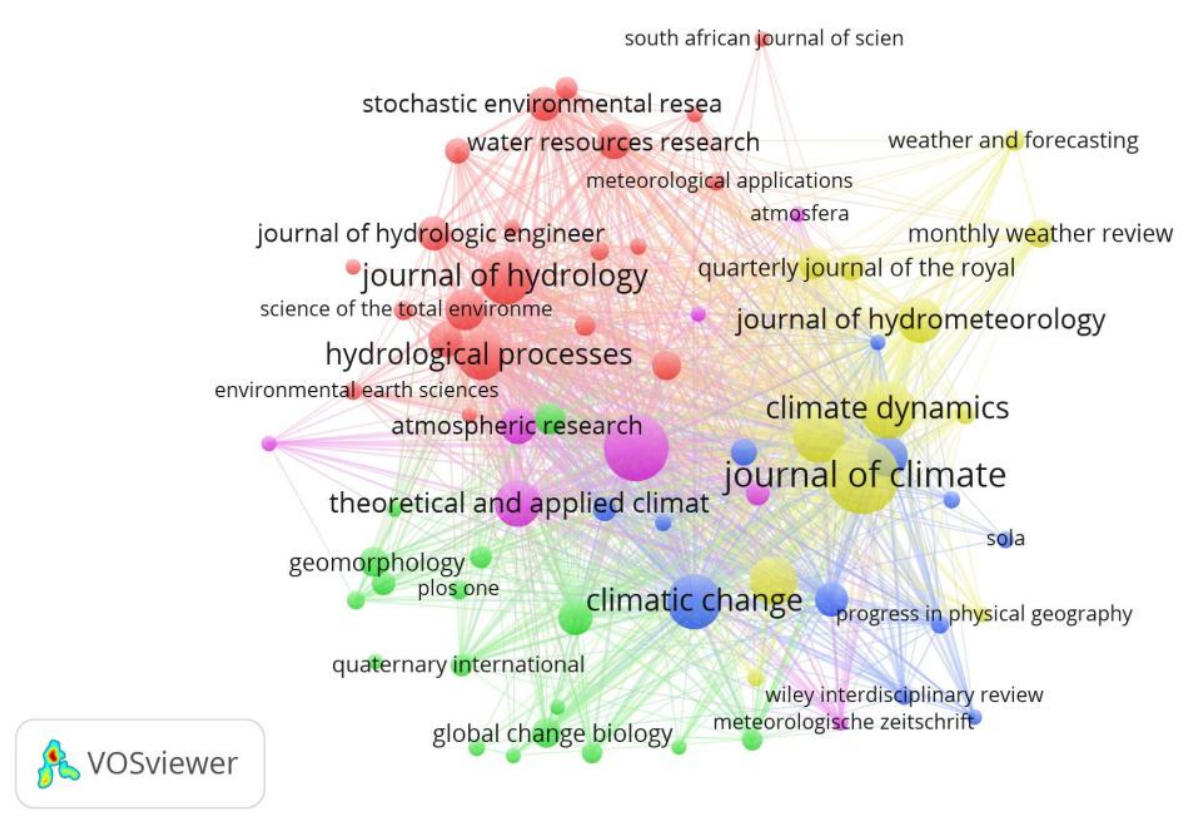

Figura 3: Mapa de acoplamento bibliométrico de periódicos. Fonte: Web of Science, 2017.

A co-citação mostra a frequência na qual dois elementos são citados juntos, com a identificação e conexão dos autores mais importantes. $\mathrm{Na}$ figura 4 , a proximidade dos clusters aponta forte relação dos autores que se destacam.

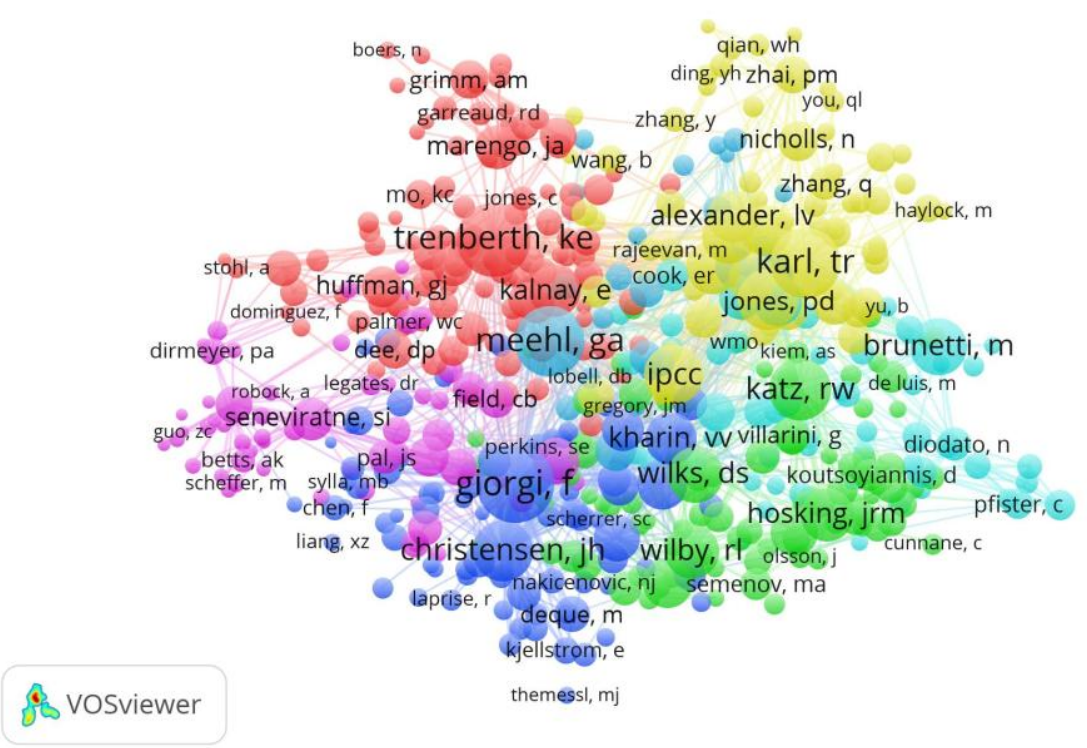

Figura 4: Mapa bibliométrico de co-citação de autores. Fonte: Web of Science, 2017. 
Com o método da análise de citação, foi possível estabelecer os trabalhos mais citados. Este método tem como objetivo indicar os principais artigos citados e quais são as tendências relevantes dentro de determinada área. A figura 5 representa o mapeamento bibliográfico destes artigos; a publicação intitulada "Climate Extremes: Observations, Modeling, and Impacts" (EASTERLING et al, 2000) obteve, no período estudado, o maior número de citações (1794 citações no total). Nota-se que, tanto no método de citação quanto no acoplamento bibliográfico, este artigo aparece em posição de destaque.

\section{\& vosviewer}

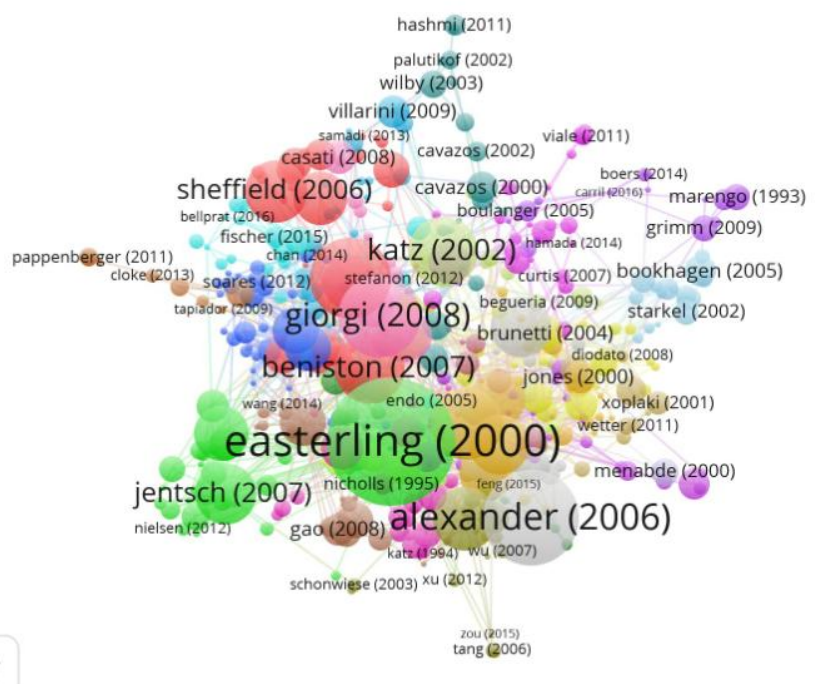

Figura 5: Mapa bibliométrico de artigos mais citados. Fonte: Web of Science, 2017.

A distribuição da co-ocorrência de palavras-chave foi reunida em seis clusters (figura 6). Cada cluster reúne palavras-chaves referentes a um tema: o cluster verde reúne palavras relacionadas à geomorfologia e hidrologia (runoff, soil erosion); o cluster vermelho agrupa termos ligados à climatologia (precipitation, storms); o cluster azul é responsável pelas palavras referentes à variabilidade climática (el niño, variability); no cluster 4 encontram-se expressões pertinentes à estatística (reanalysis, dataset) e por fim, os clusters 5 e 6 agregam palavras ligadas às mudanças climáticas (climate change, trends). A proximidade dos agrupamentos indica forte relação entre as palavras-chaves e permite identificar que a produção científica de trabalhos sobre precipitações e eventos extremos está atrelada às pesquisas sobre mudanças climáticas e com o uso de análises estatísticas. 


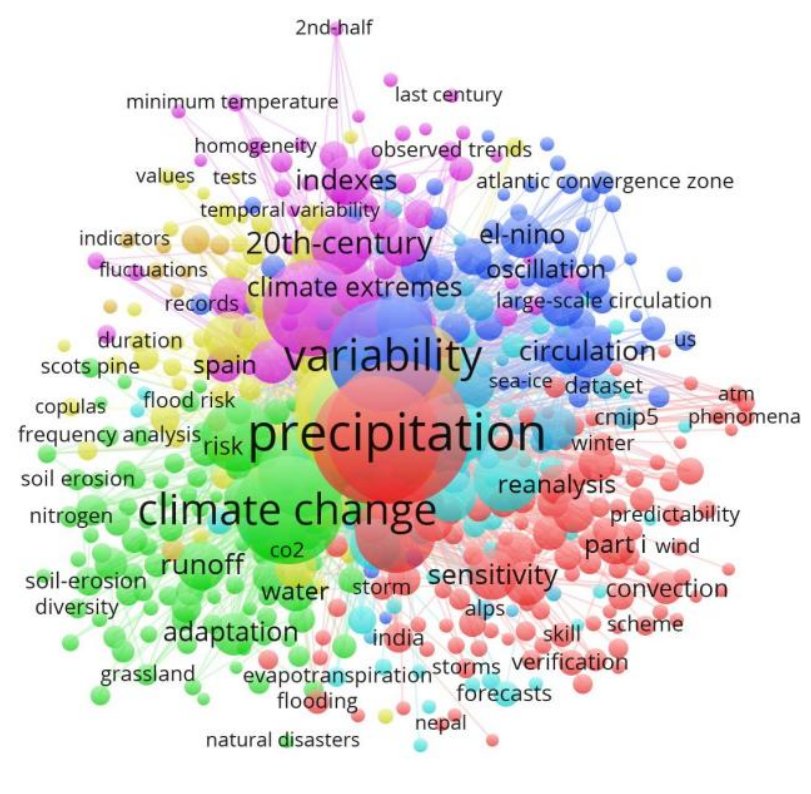

Figura 6: Mapa bibliométrico de co-ocorrência de palavras-chave. Fonte: Web of Science, 2017.

Para a definição de termos mais citados por co-ocorrêcia, foram selecionados aqueles que constam nos títulos e resumos das publicações (figura 7). A tabela I ilustra os dez termos mais presentes, que corrobora com as demais análises sobre como as palavras-chave se relacionam com os trabalhos que abordam a variabilidade, tendência e mudanças climáticas. 


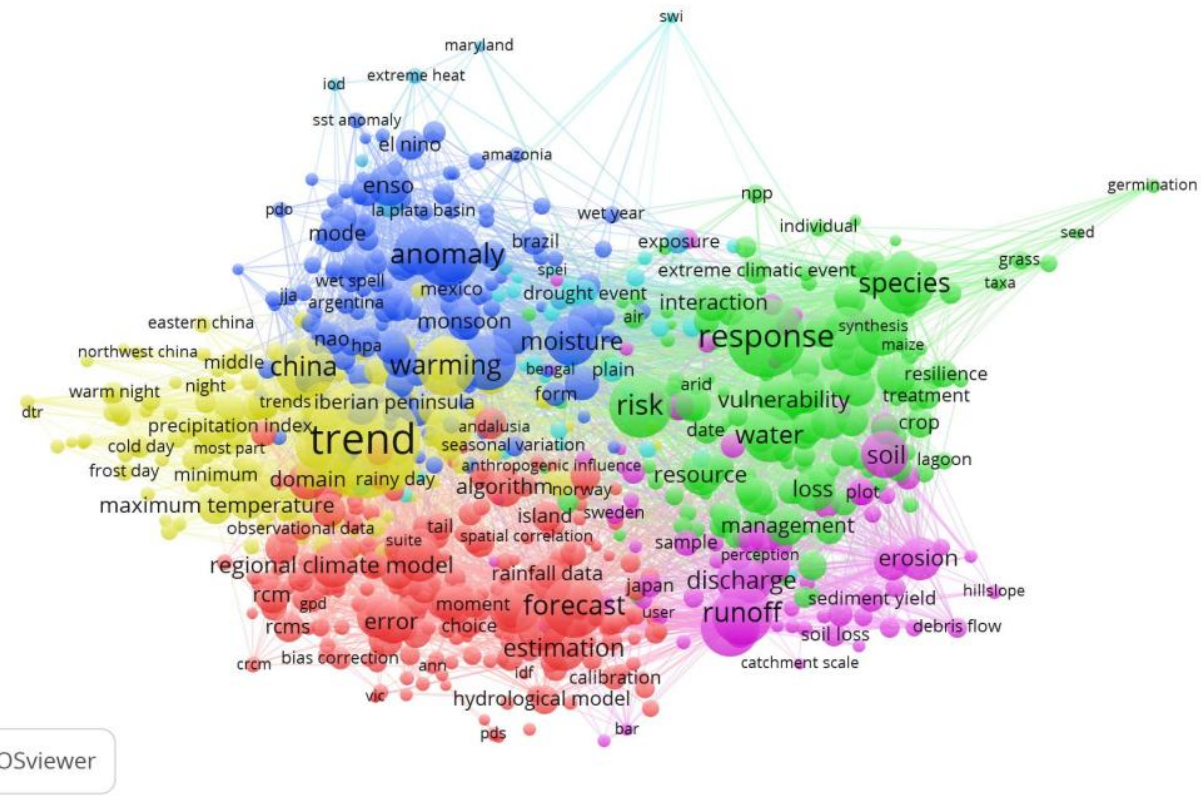

Figura 7: Mapa bibliométrico da co-ocorrência dos termos mais citados nos títulos e resumos dos artigos. Fonte: Web of Science, 2017.

Tabela I: Os dez termos mais citados. Fonte: Web of Science, 2017.

\begin{tabular}{|c|c|c|}
\hline & Termo & Número de Ocorrências \\
\hline $\mathbf{1}^{\mathbf{0}}$ & trend (tendência) & 885 \\
\hline $\mathbf{2}^{\mathbf{o}}$ & response (resposta) & 421 \\
\hline $\mathbf{3}^{\mathbf{0}}$ & anomaly (anomalia) & 281 \\
\hline $\mathbf{4}^{\mathbf{0}}$ & risk (risco) & 269 \\
\hline $\mathbf{5}^{\mathbf{0}}$ & forecast (previsão) & 255 \\
\hline $\mathbf{6}^{\mathbf{0}}$ & china (China) & 246 \\
\hline $\mathbf{7}^{\mathbf{0}}$ & runoff (escoamento) & 245 \\
\hline $\mathbf{8}^{\mathbf{0}}$ & species (espécies) & 234 \\
\hline $\mathbf{9}^{\mathbf{0}}$ & warming (aquecimento) & 234 \\
\hline $\mathbf{1 0}^{\mathbf{0}}$ & catchment (bacia/represa) & 233 \\
\hline
\end{tabular}

*Tradução dos termos realizada pelos autores.

\section{Considerações Finais}

O mapeamento bibliométrico contribuiu para o conhecimento da produção bibliográfica internacional com a combinação das palavras-chave "chuva", "precipitação" e "eventos extremos". Permitiu estabelecer quantitativamente os autores, artigos, termos e periódicos de maior expressão. Os resultados mostraram o acoplamento bibliográfico e a co-citação são técnicas que permitem estabelecer as ligações entre 


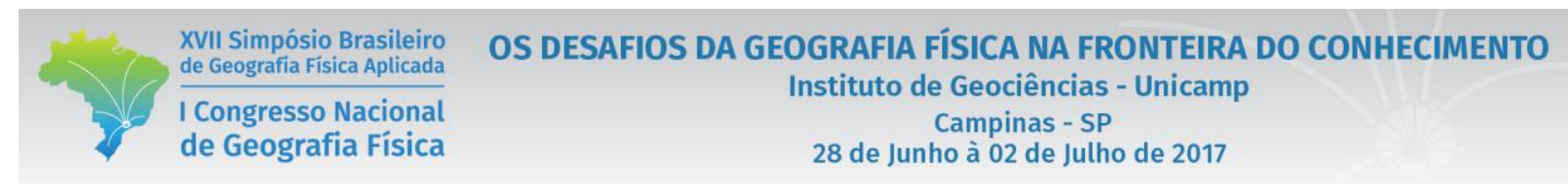

autores/artigos citantes e citados, de forma que seja possível estabelecer uma rede de áreas do conhecimento e linhas de pesquisa que são dominantes nesta temática.

As palavras-chaves com maior número de citações relacionam-se com diversas áreas do conhecimento, associadas à estatística, variabilidade e mudanças climáticas.

Essa estratégia de levantamento de artigos internacionais se mostrou ainda mais pertinente face à magnitude do tema de pesquisa. A ocorrência de eventos extremos pode desencadear desastres naturais de grande escala e conhecer o funcionamento do sistema é de suma importância para promover ações de prevenção e mitigação, de maneira que as consequências sejam minimizadas.

\section{Bibliografia}

ASSOCIAÇÃO BRASILEIRA DE NORMAS TÉCNICAS. NBR 6028: informação e documentação: resumo apresentação. Rio de Janeiro, 2003c. 2 p.

CAVALCANTI, I. F. A. Large scale and synoptic features associated with extreme precipitation over South America: A review and case studies for the first decade of the 21st century. Atmospheric Research, v. 118, p. 27-40, Nov 15 2012. ISSN 0169-8095. Disponível em: < <Go to ISI>://WOS:000310386100004 >.

EASTERLING, D. R. et al. Climate Extremes: Observations, Modeling, and Impacts. Science, v. 289, n. 5487, p. 2068-2074, 2000.

GALVANI, E.; PEREIRA, A. R. ; KLOSOWSKI, E. S. Relações entre o Índice de Oscilação Sul (IOS) e o total mensal de chuva em Maringá-PR. Acta Scientiarum (UEM) (Cessou em 2002), Maringá-Pr, v. 20, n.4, p. 531-535, 1998.

GRÁCIO, M.C.C. Acoplamento bibliográfico e análise de cocitação: revisão teórico-conceitual. Encontros Bibli: revista eletrônica de biblioteconomia e ciência da informação, v. 21, n. 47, p. 82-99, set./dez., 2016.

HAUNSCHILD, R.; BORNMANN, L.; MARX, W. (2016) Climate Change Research in View of Bibliometrics. PLoS ONE 11(7): e0160393. doi:10.1371/journal.pone.0160393.

JIANG, H.; QIANG, M.; LIN, P. A topic modeling based bibliometric exploration of hydropower research. Renewable and Sustainable Energy Reviews, v. 57, p. 226-237, 5// 2016. ISSN 1364-0321. Disponível em: < http://www.sciencedirect.com/science/article/pii/S1364032115015774 >.

KNUTAS, A., HAJIKHANI, A., SALMINEN, J., IKONEN, J., PORRAS, J. Cloud-Based Bibliometric Analysis Service for Systematic Mapping Studies. CompSysTech 2015.

ROJAS-SOLA, J. I.; AGUILERA-GARCÍA, Á. I. Análisis bibliométrico mundial de la categoría 'remote sensing' de la web of science (1997-2012). Boletim de Ciências Geodésicas, v. 20, p. 855-878, 2014. ISSN 1982-2170. Disponível em: < http:/www.scielo.br/scielo.php?script=sci_arttext\&pid=S1982$21702014000400855 \&$ nrm $=$ iso $>$.

WALTMAN, L.; VAN ECK, N. J.; NOYONS, E. C. M. A unified approach to mapping and clustering of bibliometric networks. Journal of Informetrics, v. 4, n. 4, p. 629-635, 10// 2010. ISSN 1751-1577. Disponível em: < http://www.sciencedirect.com/science/article/pii/S1751157710000660 >. 


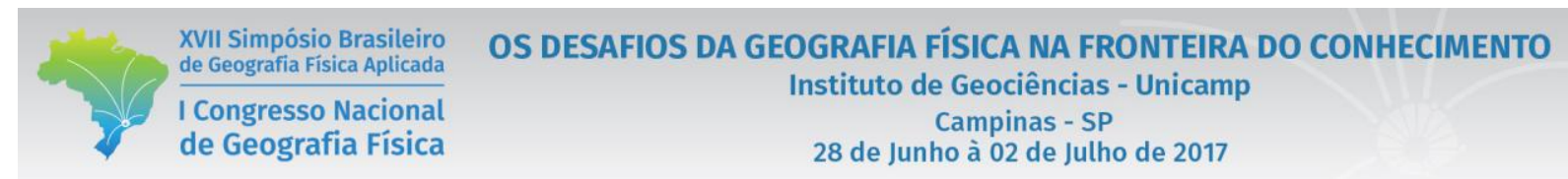

ZHANG, K. et al. A bibliometric analysis of research on carbon tax from 1989 to 2014. Renewable and Sustainable Energy Reviews, v. 58, p. 297-310, 5// 2016. ISSN 1364-0321. Disponível em: < http://www.sciencedirect.com/science/article/pii/S1364032115014720 >. 